\section{Identification of hooded rats using photographs of emerging fur patterns}

\section{CHARLES A. GRAESSLE \\ University of Illinois, Champaign, Illinois 61820}

Longitudinal observations on multiple-birth animals usually begin only after individuals grow large enough for ear-punching or toe-clipping. The procedure described by Geller and Geller (1966) is one of the only techniques ideally suited for identification of individuals in recently born litters. Their technique involves the injection of a small amount of India ink under one or more soles and palms of the newborn animals. However, even this procedure may entail more pain than desirable in studies where early experience is a variable. Painting the skin of the newborn with various colored chemicals is certainly less aversive, but this technique is sometimes not as permanent as other procedures and may produce visual or odor cues which could modify the behavior of the mother toward her young.

A permanent nonintrusive procedure is available for animals possessing unique fur markings, such as Dutchbelted rabbits and hooded rats. As exemplified in Figure 1, an individual rat's fur pattern is unique, unchanging, and discernible as early as 3 days postpartum.

Requests for reprints should be sent to: Charles Graessle, Department of Psychology, University of Illinois, Champaign, Illinois 61820. This work was supported in part by Research Grant NE-G-00-3-0013 from the National Institute of Education awarded to S. W. Porges.
The most frequent bases for identification include: (1) length of back stripe; (2) thickness of back stripe; and (3) the number of marks, protrusions, or indentations on each side of the back stripe. However, any physical characteristic may be used for identification, such as extremeiy small size or absence of a tail.

\section{PROCEDURE}

The fur pattern of each animal can be sketched or photographed. Each of these procedures can be completely nonintrusive except for whatever handling is required to orient the individual so that the back is visible. Photography is preferred because it allows the investigator to work more quickly with the animal and to make additional observations on gross anatomy (e.g., length of body) at his convenience.

If photography is chosen, a single-lens reflex camera is secured to a tripod or copy stand and focused down to a point approximately $3 / 8 \mathrm{in}$. above the table. The camera should be close enough to provide adequate detail but far enough away such that placement of the pup under the lens is not too critical. A field approximately $10 \times 15 \mathrm{~cm}$ works well for rat pups and can be obtained with a $50-\mathrm{mm}$ lens mounted on a $35-\mathrm{mm}$ camera, fitted with a No. 3 closeup lens. The edges of the field on the table are marked beforehand with masking tape while looking through the viewfinder. This eliminates the need to look through the viewfinder every time a new pup is placed under the camera.

Flourescent or incandescent illumination adequately exposes most black-and-white films. Incandescent

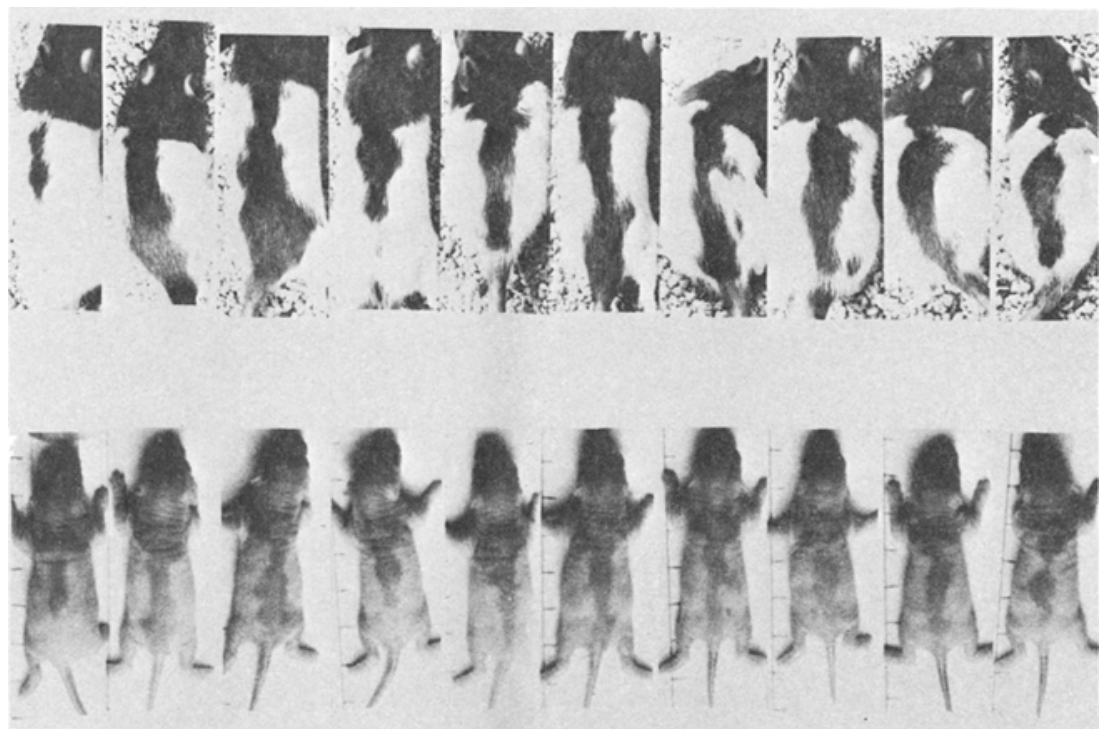

Figure 1. Fur patterns of a litter of 10 hooded rats at 3 days postpartum (left) and the same individuals at 90 days of age (right). 
lamps should be limited to $100 \mathrm{~W}$ or less and located at least $25 \mathrm{~cm}$ from the subject. If heat stress must be avoided, consider strobe flash or flourescent illumination. Fast film, such as Kodak's Tri-X, should be used. A diaphragm setting of at least $F 3.5$ is needed in order to obtain a comfortable depth of field. Three-day-old rat pups will not crawl away from the camera, but they may squirm into a comfortable position for several seconds after being placed under the camera. Shutter speeds as slow as $.25 \mathrm{sec}$ may be used, provided the shutter is operated after the animal has ceased its initial movement.

It is very important to place light directly above and to the rear of the planned location of the subject. If the back of the pup is unevenly lit, the resulting shadows will sometimes be indistinguishable from dark fur markings.

Wallet-sized enlargements of offspring from the same litter may be placed on a sheet of enlarging paper for easy selection of individuals from their littermates. Enlarging easels such as the Saunders PR810 or the Premier Multiprint Model CE-3 expedite this process.

This procedure does not allow differentiation of albino rats. Should two or more albinos appear in a hooded litter, they must be identified by other methods if sex or gross anatomy does not differentiate them. In addition, treatments that severely retard physiological growth can delay initial appearance of the fur pattern.

\section{REFERENCE}

Geller, L. M., \& Geller, E. H. A simple technique for the permanent marking of newborn albino rats. Psychological Reports, 1966, 18, 221-222.

(Received for publication February 3, 1977; revision accepted November $15,1977$. 